\title{
DYNAMICS OF PSEUDO-RADIOACTIVE CHEMICAL PRODUCTS VIA SAMPLING THEORY
}

\author{
JUAN LUIS GARCÍA GUIRAO ${ }^{1}$ AND MARÍA T. DE BUSTOS ${ }^{2}$
}

\begin{abstract}
The decomposition of radioactive chemical products usually follows an exponential dynamics. The present paper deals with the problem of analyzing the unknown dynamics of pseudo-radioactive materials for which we have a temporal sample of the amount of the decomposing product. The strategy for studying if the adjustment of the dynamics is or not of exponential type is to use a recent generalization of the Shannon's sampling theorem for non-band limited signals. The aim of the paper is to present an alternative and short proof of this result with a completely different approach to the original one by using transform theory.
\end{abstract}

\section{INTRODUCTION AND STATEMENT OF THE MAIN RESUlT}

It is well known that the dynamics of the decomposition of the most used radioactive chemical products like Carbon 14 or Urania 238 evolves in an exponential way. Imagine that we have a pseudo-radioactive material with an unknown decomposition dynamics but for which we have a temporal sample of the amount of the decomposing product. In this setting for analyzing if the dynamics has or not an exponential adjustment is used a recent generalization of the Shannon Sampling Theorem for non-band limited signals [1]

A central result of the signal theory in engineering is the well-known Shannon-Whittaker-Kotel'nikov's theorem (see for instance [11] or [13]) working for band-limited maps of $L^{2}(\mathbb{R})$ (i.e., for Paley-Wiener signals),

Key words and phrases. Pseudo-radioactive, Band-limited signal, Shannon's sampling theorem, Approximation theory.

2000 Mathematics Subject Classification. Primary 41A60, 41A46; Secondary 41A30, 41A 45, 41A58, 42C10.

This work has been partially supported by MCI (Ministerio de Ciencia e Innovación) and FEDER (Fondo Europeo Desarrollo Regional), grant number MTM200803679/MTM, Fundación Séneca de la Región de Murcia, grant number 08667/PI/08 and JCCM (Junta de Comunidades de Castilla-La Mancha), grant number PEII090220-0222. 
and based on the normalized cardinal sinus map $\operatorname{sinc}(t)$ defined by

$$
\operatorname{sinc}(t)= \begin{cases}1 & \text { if } t=0 \\ \frac{\sin (\pi t)}{\pi t} & \text { if } t \neq 0 .\end{cases}
$$

Another philosopher's stone of the signal processing theory is the Middleton's sampling theorem for band step functions (see [10]). This result was one of the first modifications of the classic Sampling theorem (see [12]) which only works for band-limited maps. After this starting point many different extensions and generalizations of this theorem appeared in the literature trying to obtain approximations of non band-limited signals (see for instance [4] or [6]). Good surveys on these extensions are [5] or [13].

[1] following the spirit of the previous results in the sense of trying to obtain approximations of non band-limited signals by using bandlimited ones by increasing the band size. But this approach is completely different to the previous ones in the sense that we keep constant the sampling frequency generalizing in the limit the results of Marvasti et al. [9] and Agud et al. [2] .

In this setting, [1] states and proof for Gaussian signals the following asymptotic Shannon sampling theorem type where the convergence is considered in the Cauchy's principal value for the series and point wise for the limit.

Property 1. Let $f: \mathbb{R} \rightarrow \mathbb{R}$ be a map and $\tau \in \mathbb{R}^{+}$. We say that $f$ holds the property $\mathcal{P}$ for $\tau$ if

$$
f(t)=\lim _{n \rightarrow \infty}\left(\sum_{k \in \mathbb{Z}} f^{\frac{1}{n}}\left(\frac{k}{\tau}\right) \operatorname{sinc}(\tau t-k)\right)^{n} .
$$

Theorem 1. The Gaussian maps, i.e. maps of the form $e^{-\lambda t^{2}}, \lambda \in \mathbb{R}^{+}$ hold property $\mathcal{P}$ for every given $\tau \in \mathbb{R}^{+}$.

The idea of the proof presented in [1] is the following: since the Gaussian map is analytical, for proving formula (1) is enough to show the equality between the coefficients of the power series representation of the Gaussian map and the coefficients of the series stated in the second member of (1) after proving the analitycity of the second member of (1). This proof is very long, quite technical and use strongly complex analysis tools.

The aim of the present paper is to present an alternative proof to this result very short and using a completely different approach. The ideas of our proof are to use some simple facts of the transform theory and to use properly a bound given by Boas [3]. 
The paper is structured as follows in Section 2 we present the ideas and results that have inspired property $\mathcal{P}$ and in Section 3 we give the new proof of Theorem 1.

\section{ON THE PROPERTY $\mathcal{P}$}

Property $\mathcal{P}$ is an approximation in the limit, through potentials of band-limited maps of the original signal, based on [2] and [9].

In [2] is proven that given a sequence $\left\{s_{k}\right\}_{k \in \mathbb{Z}} \in l^{2 / n}(\mathbb{Z}), B>0$, $\tau \geq 2 B$ and $n$ odd, there exist exactly $n$ band-limited signals $\left\{x_{r}\right\}$ with bandwidth equal to $B$ such that $x_{r}^{n}\left(\frac{k}{\tau}\right)=s_{k}$. Moreover, is shown that $x_{r}=e_{r} x_{0}$, where $\left\{e_{r}\right\}_{r=0}^{n-1}$ are the roots of unity of order $n$ and $x_{0}(t)=\sum_{k \in \mathbb{Z}} s_{k}^{1 / n} \operatorname{sinc}(2 B t-k)$.

From this is directly deduced that if we consider an odd number $n$ and a band-limited signal $f$ with bandwidth $\widetilde{B}$ such that the sequence of coefficients $\left\{f\left(\frac{k}{\tau}\right) ; k \in \mathbb{Z}\right\}$ with $\tau \geq \frac{2 \widetilde{B}}{n}$ holds the properties stated in [2], then the signal admits a recomposition of Shannon type in the form

$$
f(t)=\left(\sum_{k \in \mathbb{Z}} f^{\frac{1}{n}}\left(\frac{k}{\tau}\right) \operatorname{sinc}(\tau t-k)\right)^{n},
$$

where clearly the sampling frequency can be choosen bigger than the Nyquist one.

The aim is to provide a method for approximating non band-limited signal by band-limited ones and keeping the frequency of the sampling constant. And the idea is to take limits in (2) obtaining an equality of the form

$$
f(t)=\lim _{n \rightarrow \infty}\left(\sum_{k \in \mathbb{Z}} f^{\frac{1}{n}}\left(\frac{k}{\tau}\right) \operatorname{sinc}(\tau t-k)\right)^{n},
$$

expressed as a property $\mathcal{P}$.

\section{Reproving TheOREM 1}

The aim of this section is to proof Theorem 1 with a completely different approach to the original one.

Proof of Theorem 1.

Boas [3] states the following estimation

$$
\left|f(t)-\sum_{k} f(k) \operatorname{sinc}(t-k)\right| \leq 2 \int_{|\xi|>1 / 2}|\widehat{f}(\xi)| d \xi .
$$


We assume, without loss of generality, that $\lambda=\tau=1$. Let $f(t)=$ $e^{-\pi t^{2}}$, for which $f_{n}(t)=f^{1 / n}(t)=e^{-\pi t^{2} / n}$ with $\widehat{f}_{n}(\xi)=\sqrt{n} e^{-\pi \xi^{2} n}$. By $(3)$

$$
\begin{gathered}
\left|f^{1 / n}(t)-\sum_{k} f^{1 / n}(k) \sin c(t-k)\right| \leq 2 \sqrt{n} \int_{|\xi|>1 / 2} e^{-\pi \xi^{2} n} d \xi=(x=\xi \sqrt{n}) \\
=2 \int_{|x|>\frac{\sqrt{n}}{2}} e^{-\pi x^{2}} d x=O\left(\frac{1}{\sqrt{n}} e^{-\pi n / 4}\right)
\end{gathered}
$$

where we have used the trivial estimation

$$
\alpha \int_{x \geq \alpha} e^{-\pi x^{2}} d x \leq \int_{x \geq \alpha} x e^{-\pi x^{2}} d x=O\left(e^{-\pi \alpha^{2}}\right)
$$

Thus, writing $a_{n}=\sum_{k} f^{1 / n}(k) \sin c(t-k), b_{n}=f^{1 / n}(t)$, we have proved

$$
\left|b_{n}-a_{n}\right| \leq C \frac{1}{\sqrt{n}} e^{-\frac{\pi}{4} n}
$$

Now, using the Middle Value Theorem is

$$
\left|f(t)-a_{n}^{n}\right|=\left|b_{n}^{n}-a_{n}^{n}\right| \leq\left|b_{n}-a_{n}\right| n\left(1+\left|b_{n}-a_{n}\right|\right)^{n-1}
$$

Clearly

$$
\left(1+\left|b_{n}-a_{n}\right|\right)^{n-1} \leq\left(1+\frac{C}{\sqrt{n}} e^{-\frac{\pi}{4} n}\right)^{n-1}=O(1)
$$

since

$$
(n-1) \log \left(1+\frac{C}{\sqrt{n}} e^{-\frac{\pi}{4} n}\right) \sim n \frac{C}{\sqrt{n}} e^{-\frac{\pi}{4} n} \rightarrow 0
$$

when $n$ goes to infinity.

Therefore

$$
\left|f(t)-a_{n}^{n}\right| \leq K n\left|b_{n}-a_{n}\right| \leq K n \frac{1}{\sqrt{n}} e^{-\frac{\pi}{4} n} \rightarrow 0
$$

when $n \rightarrow \infty$ proving the result and moreover the uniform convergence under all compact set of $\mathbb{R}$.

\section{REFERENCES}

[1] A. Antuña, J.L.G. Guirao and M.A. López, An asymptotic sampling recomposition theorem for Gaussian signals, Med. J. Math., To appear.

[2] L. Agud and R.G. Catalán, New Shannon's sampling recomposition, Rev. Acad. Ciencias Zaragoza, 56 (2001), 45-48.

[3] R.P. Boas JR., Summation formulas and band-limited signals Tohoku Math. J., 24, (1972), 121-125. 
[4] P.L. Butzer, S. Ries And R.L. Stens, Approximation of continuous and discontinuous functions by generalized sampling series, Jour. Appr. Theo., 50, (1987), 25-39.

[5] P.L. Butzer And R.L. Stens, Sampling theory for not necessarily band-limited functions: a historical overview, SIAM review, 34, 4, (1992), 40-53.

[6] J.A. Gubner, A new series for approximating Voight functions, Jour. Phys. A: Math., 27, (1994), L745-L749.

[7] J.R. Higgings, Five short stories about the cardinal series, Bull. Amer. Math. Soc., 12 (1985), 45-89.

[8] H.J. Landau, H.O Pollak Prolate spheroidal wave functions, Fourier analysis and uncertainly, Bell. Sys. Tech. Jour., 40, 1, (1961), 65-84.

[9] F. MARVASTI AND A.K. JAIn, Zero crossing bandwidth compression, and restoration of nonlinearly distorted band-limited signals, J. Optical Soc. Amer., 3 (1986), 651-654.

[10] D. Middleton, An introduction to statistical communication theory, McGrawHill, New York, 1960.

[11] C.E. Shannon, Communication in the presence of noise, Proc. IRE, 137, (1949), 10-21.

[12] E.T. Whittaker, On the functions which are represented by the expansions of the interpolation theory, Proc. Roy. Soc. Edinburgh, 35, (1915), 181-194.

[13] A.I. Zayed, Advances in Shannon's Sampling Theory, Ed. CRC Press, (1993).

1 Departamento de Matemática Aplicada y Estadística. Universidad Politécnica de Cartagena, Campus de la Muralla, 30203-Cartagena

(Región de Murcia), Spain -Corresponding Author-

E-mail address: juan.garcia@upct.es

2 Departamento de Matemática Aplicada. Universidad de Salamanca, C/ Del Parque, 2, 37008-Salamanca (Castilla y León), Spain

E-mail address: tbustos@usal.es 